\title{
Water Chemistry and Trophic State of Seven Lakes of Mandya District, Karnataka, India
}

\author{
J. Mahadev*, N. S. Pankaja, G. Sugeetha, M. Mahadevamurthy and Nagarathnamma
}

Department of Forestry and Environmental Science, Pathology, Entomology, College of Agriculture, V.C. Farm, Mandya, Department of Forestry and Environmental Science, UAS (B), GKVK and Government Degree College for Women, Mandya, India

*Corresponding author

\begin{abstract}
A B S T R A C T
Keywords

Lakes, Physicochemical parameter, WQI, Nygaar's Index and Trophic state

Article Info

Accepted: 07 September 2020 Available Online: 10 October 2020

Lakes supply water for irrigation, drinking, fisheries etc and thus have significant economic and recreational values in order to determine impact of human activities on fresh water Ecosystem. In the present study, 7 lakes of Mandya district classified based on the trophic state of Physico-Chemical and biological, chlorophyll 'a'. Nygaar's trophic state and water quality index (WQI). Each objects can be simplified by the correlation matrix and attempts to distinguish logical groupings, phosphate, chlorophyll 'a', and physic chemical parameter. The correlation indicates that all lakes are Oligotrophic in nature and the most significant parameter was Chlorophyll 'a' and Phosphorus. Trophic state index based on Phosphorous in 6 lakes indicated that the category of oligotrophic lakes tending to become mesotrophic and one lake indicated that only oligotrophic. If the lakes were classified on the basis of Nygaar's trophic state index indicates that 6 lakes were in a state of oligotrophism and were fast tending become Eutrophic. The Water quality index (WQI) developed for rating of water are indicate 6 lakes were far from being pure and are unsustainable for drinking purpose except Thonnur lake. This study indicated that ideal means by which progress integrated water resources management can be monitored. Thus, the data of this findings can be used for the scientific management and restoring polluted lakes and its eutrophication.
\end{abstract}

\section{Introduction}

The monitoring of water quality especially in the inland water bodies, aquifers, lakes reservoir is important as it helps the management of the Eutrophication and productivity of the water bodies, Carlson made early attempts to define an index, termed trophic state index (TSI) which could tell about the trophic states and nature of the lake. Based on this trophic index, further classification of eutrophication of a lake into oligotrophic, mesotrophic and eutrophic were made. Because of water pollution and contamination of inland water bodies, it has become important to develop methods to determine index of eutrophication of lakes that provide better spatial and temporal coverage. During recent year large number of publications have been appeared on fresh 
water regarding its suitability for domestic as well as non-domestic purpose, similar work done by Hosmani and Bharath (1980), Hosmani and Vasanthkumar (1996), Mahadev et al., (2004). The present work was undertaken with the objective of elucidating lake water and establishing interrelationship between various Physio-chemical parameter including, Sakamoto index, EPA NEW trophic index, Carlsons trophic state index, Nygaards algal index and water quality index (WQI). Based on Physico-chemical parameter Chlorophyll 'a' and phosphorous of 7 lakes were studied. This study indicate that ideal means by which progress integrate water resource management can be monitored.

\section{Materials and Methods}

A Mandya district is situated in the southern part of Karnataka state and lies between $76^{\circ}$ $19^{\prime}$ and $77^{\circ} 22^{\prime}$ 'east longitude and $12^{\circ} 13^{\prime}$ and $13^{0} 04^{\prime}$ north latitude. It receives an average annual rainfall of about $180 \mathrm{~mm}$ and most part of the land is irrigated by huge channels. The district also has several small and large reservoirs composite. Water samples were collected and analyzed from each of 7 lakes covering most part of the district for the period from March 2016 to February 2018. A brief description of these lakes are presented in Table 1. The methods followed for the determination of Physico-chemical parameter. APHA (2002), Trivedy and Goel (1986), Hosamani and Vasanthkumar (2002), Mahadev, et al., (2004), The collection preservation and enumeration of phytoplanktons were all as described by Munnawar (1970), Hutchinson (1967), Patrick (1973), Prescott (1982), Jones et al., (1993), Kaloo et al., (1995) Seemajohori et.al (1990). In the present analysis Chlorophyll ' $a$ ' $\mathrm{NO}_{2}-\mathrm{N}$ phosphorus and SDD have been chosen as the potential candidates for estimating of TSI which can cover range concentration of each of three parameters and applicable to any lake, Chlorophyll 'a' are representation of the optical scattering and absorption in the lake water. The light availability in the controls the general growth and health of microorganism, biomass, flora and nitrogen is one of the major nutrient components of the food chain of lakes. It lies at the base of food web and provide food for all living organism.

\section{Results and Discussion}

A data collected over two years during monsoon, summer and pre monsoon provided a basis of the results in the study during period March 2016 to February 2018. The data contains large variability. In the present study, 7 lakes of Mandya district classifying the trophic state based on Physio-Chemical, Chlorophylla, Phosphorus, Nygaars tropic state and water quality index (WQI) were represented in Table 2 to $5 \mathrm{C}$.

\section{Classification of Lakes}

The various methods have been adopted for classification of lake waters and to indicate their trophic status.

The value of Chlorophyll 'a' and Phosphate indicated that all lakes range from Oligotrophic to Mesotrophic nature According to sakamoto classification all 7 lakes fall under the categories of oligo trophic lake and tend to become mestrophic except Thonnur Lake was purely oligotrophic and Konanahallilake tend to became Mesotrophic to Eutrophic.

EPA and NEW trophic state index Guthalu lake, Sathanur Lake, Dodda Lake, Sullekere Lake indicated that oligotrophic to tend become mesotrophic, Thonnur lake (2.03) shows purely oligotrophic, Konanahalli lake (10.36) under categories of mesotrophic to eutrophic. 


\section{Based on Carison (1977)}

According to this classification Chlorophyll 'a' and Phosphorous among in 7 lakes 6 lakes fall under the category of oligotrophic lakes and tend to become mesotrophic except Thonnur lake purely become oligotrophic in throughout study period and Konanahalli lake is mesotrophic, tending to eutrophic

Table.1 Physical feature of 7 lakes of Mandya District

\begin{tabular}{|c|c|c|c|c|c|c|c|}
\hline \multicolumn{2}{|r|}{ Lakes } & Location & Catchment & Depth & Soil & Micro & Usage \\
\hline 1 & Guthalu Lake & $\begin{array}{l}\text { Longitude }-76^{0} 50^{\prime} \mathrm{E} \\
\text { Latitude }-12^{0} 30^{\prime} \mathrm{N}\end{array}$ & 60.00 & 7.0 & RSL & $a, b, c$ & $\begin{array}{l}\text { Irrigation, } \\
\text { agriculture, } \\
\text { domestic }\end{array}$ \\
\hline 2 & Konanahalli Lake & $\begin{array}{l}\text { Longitude }-76^{0} 45^{\prime} \mathrm{E} \\
\text { Latitude }-12^{0} 30^{\prime} \mathrm{N}\end{array}$ & 36.00 & 10.00 & RSL & $\mathrm{a}, \mathrm{c}, \mathrm{d}$ & $\begin{array}{l}\text { Domestic, } \\
\text { Agriculture }\end{array}$ \\
\hline 3 & Thonnur Lake & $\begin{array}{l}\text { Longitude }-76^{0} 38^{\prime} \mathrm{E} \\
\text { Latitude }-12^{0} 34^{\prime} \mathrm{N}\end{array}$ & 311.00 & 18.00 & RSL & Nil & $\begin{array}{l}\text { Agriculture, } \\
\text { recreation }\end{array}$ \\
\hline 4 & Sathanur Lake & $\begin{array}{l}\text { Longitude }-76^{0} 47^{\prime} \mathrm{E} \\
\text { Latitude }-12^{0} 54^{\prime} \mathrm{N}\end{array}$ & 30.00 & 8.00 & RSL & $\mathrm{c}, \mathrm{e}$ & $\begin{array}{l}\text { Agriculture, } \\
\text { Domestic, } \\
\text { Agriculture }\end{array}$ \\
\hline 5 & $\begin{array}{ll}\text { Dodda } & \text { Lake } \\
\text { (Malavalli) } & \end{array}$ & $\begin{array}{l}\text { Longitude }-77^{0} 20^{\prime} \mathrm{E} \\
\text { Latitude }-13^{0} 45^{\prime} \mathrm{N}\end{array}$ & 10.00 & 6.00 & RCL & $\mathrm{c}, \mathrm{e}, \mathrm{f}$ & $\begin{array}{l}\text { Domestic } \\
\text { Agriculture, } \\
\text { Agriculture }\end{array}$ \\
\hline 6 & Mangala Lake & $\begin{array}{l}\text { Longitude }-76^{0} \quad 50^{\prime} \quad \mathrm{E} \\
\text { Latitude }-12^{0} 30^{\prime} \mathrm{N}\end{array}$ & 47.00 & 10.00 & RSL & $\mathrm{a}, \mathrm{e}, \mathrm{k}$ & $\begin{array}{l}\text { Domestic } \\
\text { Agriculture, } \\
\text { Agriculture }\end{array}$ \\
\hline 7 & Sullekere Lake & $\begin{array}{l}\text { Longitude }-77^{0} 01^{\prime} \mathrm{E} \\
\text { Latitude }-12^{0} 33^{\prime} \mathrm{N}\end{array}$ & 70.00 & 12.00 & RSL & $\mathrm{c}, \mathrm{e}, \mathrm{d}$ & $\begin{array}{l}\text { Irrigation } \\
\text { Agriculture }\end{array}$ \\
\hline
\end{tabular}

a) Eichorrnia crassipes b) Pistialstratirles c) Typhaangustala d) Jussiacaadscondens e) Azolo

f) RSL: Redsoil and Loamy g) RCL: Red Clayey Loam

Table.2 Based on the amount of Chlorophyll 'a' and Phosphate index

\begin{tabular}{|l|c|c|c|}
\hline \multicolumn{1}{|c|}{ Trophic condition } & Sakamoto & $\begin{array}{r}\text { Academy Chlorophyll 'a' } \\
\mathbf{M g} / \mathbf{M}^{\mathbf{3}}\end{array}$ & Dibson \\
\hline Oligotrophic & $0.3-2.5$ & $0-4$ & 0.4 .3 \\
\hline Mesotrophic & $1-15$ & $4-10$ & $4.3-8.8$ \\
\hline Eutrophic & $5-140$ & $>10$ & $>8.8$ \\
\hline
\end{tabular}

Table.2a EPA NES trophic state index

\begin{tabular}{|l|l|l|}
\hline \multicolumn{1}{|c|}{ Trophic state } & \multicolumn{1}{|c|}{$\begin{array}{c}\text { Academy Chlorophyll } \\
\mathbf{M g} / \mathbf{M}^{\mathbf{3}}\end{array}$} & $\begin{array}{c}\text { Total Phosphoron } \\
\mathbf{M g} / \mathbf{M}^{\mathbf{3}}\end{array}$ \\
\hline Oligotrophic & $<7$ & $<10$ \\
\hline Mesotrophic & $7-12$ & $10-20$ \\
\hline Eutrophic & $>12$ & $>20$ \\
\hline
\end{tabular}


Table.2b Trophic condition of Chlorophyll 'a' and Total Phosphorous in 7 lakes of Mandya

\begin{tabular}{|l|l|c|c|}
\hline Lake & $\begin{array}{c}\text { Academy Chlorophyll 'a' } \\
\mathbf{M g} / \mathbf{M}^{\mathbf{3}}\end{array}$ & $\begin{array}{c}\text { Total Phosphorous } \\
\mathbf{M g} / \mathbf{M}^{\mathbf{3}}\end{array}$ \\
\hline $\mathbf{1}$ & Guthalu Lake & $6.39(\mathrm{~m})$ & 0.72 \\
\hline $\mathbf{2}$ & Konanahalli Lake & $10.36(\mathrm{~m})$ & 2.24 \\
\hline $\mathbf{3}$ & Thonnur Lake & $2.03(\mathrm{O})$ & 0.08 \\
\hline $\mathbf{4}$ & Sathanur Lake & $6.83(\mathrm{~m})$ & 0.68 \\
\hline $\mathbf{5}$ & Dodda Lake (Malavalli) & $5.22(\mathrm{~m})$ & 0.56 \\
\hline $\mathbf{6}$ & Mangala Lake & $8.21(\mathrm{~m})$ & 0.68 \\
\hline $\mathbf{7}$ & Sullekere Lake & $6.88(\mathrm{~m})$ & 0.59 \\
\hline
\end{tabular}

(M-Mesotrophic, O-Oligotrophic (Dobson classification)

Table. 3 Carisons trophic state index in 7 lakes of Mandya

\begin{tabular}{|l|l|c|c|}
\hline $\begin{array}{c}\text { SI. } \\
\text { No. }\end{array}$ & \multicolumn{1}{|c|}{ Lakes } & $\begin{array}{c}\text { Chlorophyll } \\
\mathbf{M g} / \mathbf{M}^{\mathbf{3}}\end{array}$ & $\begin{array}{c}\text { Phosphate } \\
\mathbf{M g} / \mathbf{M}^{\mathbf{3}}\end{array}$ \\
\hline $\mathbf{1}$ & Guthalu Lake & 8.64 & 0.98 \\
\hline $\mathbf{2}$ & Konanahalli Lake & 9.26 & 1.24 \\
\hline $\mathbf{3}$ & Thonnur Lake & 2.16 & 0.08 \\
\hline $\mathbf{4}$ & Sathanur Lake & 8.93 & 0.94 \\
\hline $\mathbf{5}$ & Dodda Lake (Malavalli) & 6.81 & 0.45 \\
\hline $\mathbf{6}$ & Mangala Lake & 9.22 & 0.65 \\
\hline $\mathbf{7}$ & Sullekere Lake & 7.20 & 0.54 \\
\hline
\end{tabular}

Table.3(a) Carison Trophic index in surface total phosphorus and total surface Chlorophylla ' $a$ '

\begin{tabular}{|l|l|c|c|}
\hline TSI & Carison trophic index & $\begin{array}{c}\text { Summer surface total } \\
\text { phosphorous } \\
\mathbf{M g} / \mathbf{M}^{\mathbf{3}}\end{array}$ & $\begin{array}{c}\text { Summer surface total } \\
\text { Chlorophyll 'a' } \\
\mathbf{M g} / \mathbf{M}^{\mathbf{3}}\end{array}$ \\
\hline $\mathbf{0}$ & Oligotrophic & 0.75 & 0.04 \\
\hline $\mathbf{1 0}$ & Oligotrophic & 1.50 & 0.12 \\
\hline $\mathbf{2 0}$ & Oligotrophic & 3.0 & 0.34 \\
\hline $\mathbf{3 0}$ & Oligotrophic & 6.0 & 0.94 \\
\hline $\mathbf{4 0}$ & Oligotrophic & 12.0 & 2.60 \\
\hline $\mathbf{5 0}$ & Mesotrophic & 24.0 & 6.4 \\
\hline $\mathbf{6 0}$ & Eutrophic & 48.0 & 20 \\
\hline $\mathbf{7 0}$ & Eutrophic & 96.0 & 56 \\
\hline $\mathbf{8 0}$ & Eutrophic & 192 & 154 \\
\hline $\mathbf{9 0}$ & Eutrophic & 384 & 427 \\
\hline $\mathbf{1 0 0}$ & Eutrophic & 768 & 1183 \\
\hline
\end{tabular}


Table.4 Nygarra's Index for water of Mandya district

\begin{tabular}{|l|c|c|l|}
\hline \multicolumn{1}{|c|}{ Index } & Values & Oligotrophic & \multicolumn{1}{c|}{ State } \\
\hline Myxophycean & 1.2 & - & Eutrophic \\
\hline Chlorophycean & 1.3 & - & Eutrophic \\
\hline Diatim & 0.07 & - & Oligotrophic \\
\hline Euglenophycean & 0.3 & - & Oligotrophic \\
\hline Compound Coefficient & 3.4 & - & Eutrophic \\
\hline
\end{tabular}

Table.4(a) Nygaar's algal Index in 7 lakes of Mandya

\begin{tabular}{|l|l|l|l|l|l|l|l|}
\hline \multicolumn{1}{|c|}{ Index } & \multicolumn{1}{c|}{ GL } & \multicolumn{1}{c|}{ KL } & \multicolumn{1}{c|}{ TL } & \multicolumn{1}{c|}{ SL } & \multicolumn{1}{c|}{ DL } & \multicolumn{1}{c|}{ ML } & \multicolumn{1}{c|}{ SL } \\
\hline Myxophycean & 1.4 & 2.2 & 0.4 & 1.4 & 1.3 & 1.6 & 1.5 \\
\hline Chlorophycean & 5.3 & 8.4 & 0.6 & 5.8 & 4.8 & 6.2 & 5.4 \\
\hline Diatoms & 1.64 & 1.92 & 0.3 & 1.60 & 1.43 & 1.52 & 1.24 \\
\hline Euglenophycean & 0.98 & 1.14 & 0.08 & 0.8 & 0.73 & 0.68 & 0.61 \\
\hline Compound coefficient & 1.8 & 2.8 & 0.8 & 1.64 & 1.52 & 1.44 & 1.68 \\
\hline
\end{tabular}

GL- Guthal Lake, KL-Konanahalli Lake, TL- Thonnur Lake, SL-Sathanur Lake, DL-Dodda Lake, ML-Mangala Lake, SL- Sullekere Lake

Table.4(b) Nygaards algal index

\begin{tabular}{|l|l|l|l|}
\hline \multicolumn{1}{|c|}{ Index } & \multicolumn{1}{|c|}{ Calculation } & \multicolumn{1}{c|}{ Oligtrophic } & \multicolumn{1}{c|}{ Eutrophic } \\
\hline Myxophycean & Myxophycean / desimidacea & $0.0-0.4$ & $0.1-3.0$ \\
\hline Chlorophycean & Chlorococcales / desimidacae & $0.0-0.7$ & $0.2-9.0$ \\
\hline Diatoms & $\begin{array}{l}\text { Centric diatom / Pennate diatom } \\
\text { Euglenophycean }\end{array}$ & $\begin{array}{l}\text { Euglenophyta / Myxophycean + } \\
\text { Chlorphycea }\end{array}$ & $0.0-0.3$ \\
\hline Compound coefficient & $\begin{array}{l}\text { Myxiphycean + Chlorococcaly + Centric } \\
\text { diatom + Englenophycea / desimid }\end{array}$ & $0.0-1.75$ \\
\hline
\end{tabular}

Table.5 Physico-chemical parameter and Water Quality Index (WQI) Horton 1965

\begin{tabular}{|l|l|l|l|l|l|l|l|l|}
\hline Lakes & pH & TDS & $\begin{array}{c}\text { Total } \\
\text { alkanity }\end{array}$ & $\begin{array}{c}\text { Total } \\
\text { Hardness }\end{array}$ & Chloride & DO & BOD & Sulphate \\
\hline GL & 8.3 & 520.00 & 243.00 & 380.00 & 256.00 & 3.8 & 3.2 & 64.00 \\
\hline KL & 9.0 & 680.00 & 350.00 & 320.00 & 286.00 & 2.4 & 3.4 & 86.00 \\
\hline TL & 7.14 & 175.00 & 115.00 & 176.00 & 62.00 & 6.2 & 8.4 & 0.00 \\
\hline SL & 8.14 & 512.00 & 215.00 & 326.00 & 216.00 & 4.3 & 4.8 & 58.00 \\
\hline DL & 7.98 & 531.00 & 220.00 & 308.00 & 244.00 & 4.2 & 4.8 & 64.00 \\
\hline ML & 8.13 & 543.00 & 208.00 & 326.00 & 250.00 & 3.9 & 4.9 & 49.00 \\
\hline SL & 8.15 & 560.00 & 214.00 & 316.00 & 254.00 & 4.0 & 4.8 & 52.00 \\
\hline
\end{tabular}

(All parameters were $\mathrm{Mg} / \mathrm{L}$ except $\mathrm{pH}$ )

GL- Guthal Lake, KL-Konanahalli Lake, TL- Thonnur Lake, SL-Sathanur Lake, DL-Dodda Lake, ML-Mangala Lake, SL- Sullekere Lake. 
Table.5(a) Water quality rating (qn) in 7 lakes of Mandya District

\begin{tabular}{|l|l|l|l|l|l|l|l|l|l|l|}
\hline $\begin{array}{l}\text { Sl. } \\
\text { No }\end{array}$ & Lakes & pH & TDS & $\begin{array}{l}\text { Total } \\
\text { alkalinity }\end{array}$ & $\begin{array}{l}\text { Total } \\
\text { hardness }\end{array}$ & Chloride & DO & BOD & $\begin{array}{l}\text { Sulp } \\
\text { hate }\end{array}$ & Total \\
\hline $\mathbf{1}$ & Guthalu Lake & 96.8 & 48.14 & 246.00 & 108.40 & 86.80 & 134.24 & 80.2 & 0.64 & 801.22 \\
\hline $\mathbf{2}$ & Konanahalli Lake & 98.4 & 102.28 & 356.40 & 158.54 & 68.10 & 120.40 & 108 & 0.86 & 1098.02 \\
\hline $\mathbf{3}$ & Thonnur Lake & 26.88 & 20.18 & 104.40 & 58.00 & 24.00 & 48.5 & 20.00 & 00 & 301.96 \\
\hline $\mathbf{4}$ & Sathanur Lake & 94.22 & 86.24 & 204.00 & 96.32 & 78.20 & 138.2 & 90.60 & 00 & 787.46 \\
\hline $\mathbf{5}$ & $\begin{array}{l}\text { Dodda } \\
\text { (Malavalli) }\end{array}$ & 86.66 & 40.54 & 181.42 & 88.4 & 53.96 & 94.05 & 64.00 & 0.30 & 609.37 \\
\hline $\mathbf{6}$ & Mangala Lake & 73.40 & 52.00 & 164.20 & 98.30 & 76.40 & 108.00 & 96.00 & 0.40 & 668.70 \\
\hline $\mathbf{7}$ & Sullekere Lake & 58.64 & 68.60 & 244.16 & 67.43 & 38.40 & 102.45 & 78.90 & 0.30 & 658.83 \\
\hline
\end{tabular}

Table.(5b) Drinking Water standard recommending agency $\mathrm{Mg} / \mathrm{L}$

\begin{tabular}{|l|c|c|}
\hline \multicolumn{1}{|c|}{ Parameter } & Standard & Recommending Agency \\
\hline pH & $7.0+8.5$ & ICMR \\
\hline TDS & 500 & WHO \\
\hline Total Alkalinity & 120 & ICMR \\
\hline Total Hardness & 300 & ICMR \\
\hline Chloride & 250 & ICMR \\
\hline Sulphate & 250 & WHO \\
\hline Dissolved Oxygen & 5.0 & ICMR \\
\hline BoD & 5.0 & ICMR \\
\hline
\end{tabular}

Table.(5c) Status of Water quality based on WQI

\begin{tabular}{|l|l|}
\hline \multicolumn{1}{|c|}{ WQI } & \multicolumn{1}{c|}{ Status } \\
\hline $\mathbf{0 - 2 5}$ & Excellent \\
\hline $\mathbf{2 6 - 5 0}$ & Good \\
\hline $\mathbf{5 1 - 7 5}$ & Poor \\
\hline $\mathbf{7 6 - 1 0 0}$ & Very Poor \\
\hline $\mathbf{1 0 0} \&$ above & Unsuitable for drinking \\
\hline
\end{tabular}

\section{Nygaar's algal Index}

Nygaarrad (1949) proposed 5 indices the evaluate to organic pollution of water body on the basis of occurrence of algal groups and designated them as Myxophycean Index, Chlorophycean Index, diatom Index, Euglenophycean Index, Based on algal count indices 6 lakes are Eutrophic one lakes still oligtrophic states.The physico-chemical parameters found to be high in Konanahalli lake and Sullekerelake compared to other lakes. All the physico-chemical parameters are within the permissible limit and is suitable for domestic porpose. An excellent test based on 7 parameters indicated that 6 lakes were far from being pure and were not suitable for drinking purpose. Based on 7 parameter indicated that in Thonnur lake all the parameter were within permissible limits and was suitable for drinking purpose.

\section{Shannon and Weaver Index 1949}

Species diversity Index: The order of pollution among lakes range as follows. 
Thonnur Lake had high species diversity index and was less polluted, while Konanahalli lake had least species diversity index and was highly polluted whereas rest of the lakes were moderately polluted.

In conclusion the trophic state index of 5 lakes indicated that they range from oligotrophic to mesotrophic and Konanahalli lake, has mesotrophic to eutrophic and Thonnur lake purely oligotrophic lake. Among 7 lakes 6 lakes indicated that was not suitable for drinking. Based on most significant parameters viz., Chlorophyll'a' and Phosphorous, among the 7 lakes, 6 lakes indicated that the categories of oligotrophic lake tending to become mesotrophic. The Nygaars trophic state index indicates that all the lakes are in a state of oligotrophic fast tending become eutrophic except Thonnur lakes. Water Quality Index indicates that among 7 lakes, 6 lakes not suitable for drinking purpose. Shannon and Weaver index indicate that Thonnurlake had high species diversity and less pollution. While Konanahalli lake had least species diversity and was highly polluted. Whereas, rest of the lakes were moderately polluted. The data of the findings can be used for scientific management and restoring the polluted and eutrophic status of the lake.

\section{References}

APHA, AWWA WPCF, 2002, Standard method of examination of water and waste water and sewage $\left(20^{\text {th }}\right.$ Education) Washington DC.

Hosmani, S.P. and Vasanthkumar. L., 1996, Calcium carbonate saturation index and its influence of phytoplankton. Pol. Res.,
15 (3): 285-288.

Hutchinson, 1967: A treatise on Limnology Vol.I introduction to lake biology and the Limnoplankton. John willey and Sons Inc. Newyork., P 1115

Jones, J. R., Lohman, T. and Umana, G., 1993, Water chemistry and trophic state of 8 lakes in costaricaverh. Intemat.VereinLimnol, 25: 899-905

Kaloo, Z. A., Pandit, A.K. and Zutshi, D. P., 1995, of Nutrient status and Phytoplankton dynamics of Dallake under salviniaratens an obnoxious weed growth oriental sci., 7: 73-85

Mahadev, J. Nagrathna and Hosmani, S. P., 2004, Studies on water quality with significance of calcium carbonate saturation index in two water bodies of Mysore. IJEP, 24(9): 67-68.

Munnawar, M., 1970, Limnology studies on fresh water ponds of Hyderabad India II. The Biotope, Hydrobiologia. 35: $127-$ 165.

Patrick, R. 1973, Use of algae, especially diatoms in the assessment of water quality. American society for testing and methods special technical publication, 528: 76-95

Prescott, G.W., 1982, Algae of the western great lakes area Ottokaetz science publishers W. German, PP 997

Seemajohori, Chaudhari U.S. and Chaudhari, P.R., 1990, Eutrophic status of some lotic and centric water bodies in Ameravathi district J. Ecotoxicol Evn., 9(1): 35040

Trivedy, R.K. and Goel PK (1986): Chemical and biological method for water pollution studies environ media publication Karod. PP. 209.

\section{How to cite this article:}

Mahadev, J., N. S. Pankaja, G. Sugeetha, M. Mahadevamurthy and Nagarathnamma. 2020. Water Chemistry and Trophic State of Seven Lakes of Mandya District, Karnataka, India. Int.J.Curr.Microbiol.App.Sci. 9(10): 85-91. doi: https://doi.org/10.20546/ijcmas.2020.910.012 